Cell adhesion molecules in enteropathic processes

11 Hill S M., Milla P J., Caseseca T'., Mirakian R.

Dept. of Child Health, Institute of Child Health and Dept. of Imminology University College \& Middlesex School of Medicine. The immune response is successfully activated when cells expressing $\mathrm{HLA}$ class II molecules present antigen to $\mathrm{T}$ lymphocytes, and specific "accessory" molecules also expressed on the surfaces of the cells involved to allow effective cell adhesion. Two such molecules are intercellular adhesion molecule 1 (ICAM-1) detected on antigen presenting cells which acts as a ligand for lymphocyte runction
associated antigen 1 (LFA-1) found on leucocytes. In Coeliac Disease (CD) and associated antigen 1 (LFA-1) found on leucocytes. In Coeliac Disease (CD) and
autoimmune enteropathy (AIE) aberrant class II/DR expression occurs on je junal autoimmine enteropathy (AIE) aberrant class II/DR expression occurs on jejun
crypt enterocytes. We have previously suggested that in AIE altered antigen presentation initiates and/or perpetuates the enteropathic process. We now investigate whether altered adhesion molecule expression plays a role in this process. Je junal mucosa from $?$ children with AIE, 5 with CD, and 5 controls with histologically nomal mucosa and normal DR expression was compared. Cryostat sections What ing monoclonal antiwere stained by an indirect imunot luorescence technique using monoclonal antibodies to ICAM-1, LFA-1, and HLA-DR. In both CD and AIF aberrant DR expression by crypt enterocytes was present. ICAM-1 was expressed by crypt enterocytes in al with AIE and LFA-1 in 3/7. Both ICAM-1 and LFA-1 were negative in CD and control crypt cells and in all villous enterocytes. Throughout the lamina propria of all
biopsies LFA-1 and ICAM-1 +ve cells were detected but in greater numbers with more biopsies LFA-1 and ICAM-1 +ve cells were detected but in greater numbers with more
intense staining in AIE. Thus in AIE but not CD aberrant Dit expression correlates intense staining in AIE. Thus in AIE but not CD aberrant Dit expression correlates this may facilitate autoantigen presentation by these enterocytes and so perpetuate the enteropathic process.
DESCRIPTION OF A PUTATIVE ENTEROTOXIN PRODUCED BY (EAggEC).

S.J.Savarino, A.Fasano, D.C.Robertson, M.M.Levine EAggEC have been associated with persistent diarrhea in children, yet little is known about virulence factors. Aim. We assayed for enterotoxic activity in culture filcrates(CF) of EAggEC strains. EAggEC strains and E. coli HBlol were added to stripped other ileal mucosa(RIM) mounted in Ussing chambers(UCs). CFs were also tested by E. coll STa ELISA and suckling mouse assay (SMA). EAggEC colonies were hybridized under low stringency to STh and caused a greater rise in short circuit current ( $\triangle I s c=55.2 \pm 5$. uAmp $/ \mathrm{cm} 2$ ) (Mean $\pm \mathrm{SE}$ ) than that seen in $\mathrm{HBl01}$ ( $\mathrm{Clsc}=55.2 \pm 5.3$ $\pm 3.0 \mathrm{uAmp} / \mathrm{cm} 2)(\mathrm{p}<0.001)$. Th $1 \mathrm{~s}$ activity on control (A $1 \mathrm{sc}=16.8$ after heating $\left(15 \mathrm{~m}\right.$ at $\left.65^{\circ} \mathrm{C}\right) ; 2$ ) localized to the $2-5 \mathrm{kDa} N \mathrm{~N}$ size range; and 3) reproduced with CF from HBIOl transformed with 17-2 plasmld. Compared to $\triangle \mathrm{Isc}$ response to $8-\mathrm{Br}-\mathrm{cGMP}$ on negative control tissue ( $\Delta$ Isc $=123.4 \pm 7.9 \mathrm{uAmp} / \mathrm{cm} 2$ ), the $\Delta$ Isc to $8-\mathrm{Br}-\mathrm{cGMP}$ added to t1ssue pretreated with $17-2 \mathrm{CF}(87.55 \pm 8.2 \mathrm{uAmp} / \mathrm{cm} 2)$ was significantly less $(p<0.01)$, while the additive effect of $17-2$ $\mathrm{CF}$ and $8-\mathrm{Br}-\mathrm{cGMP}(\triangle \mathrm{Isc}=142.8 \pm 9.1 \mathrm{uAmp} / \mathrm{cm} 2)$ was not significantly different $(p>0.05)$. < $10 \mathrm{kDa}$ CF fractions from $6 / 8$ other EAggEC strains screened in UCs gave $\Delta$ Isc responses similar to 17-2. The 17-2 CF activity was 1) non-neutralizable with polyclonal anti-STa in UCs; 2) non-reactive by STa E.LISA; and 3) negative In SMA. Also, EAggEC colonies did not hybridize with STh or STP DNA probes. Conclusions. EAggEC strain 17-2 produces an extracellular, low MW, partially heat stable molety which gives an electrical response in vitro consistent with an enterotoxic effect. This putative
F.AggEC heat stable enterotoxin (EAST) appears to be plasmid-medi-
ated, not genetically or Immunologically cross-reactive with E. coli STa, and may act via cGMP as intracellular mediator.

\section{Congenital chloridorrhoea (CCD) without diarrhoea!}

12

Milla P J., Bisset W M., Sanderson I., Jenkins H R.

rbspitals for Sick Children and Institute of Child Health, London.

Congenital chloridorrhoea is an inherited life threatening disonder usually with profuse watery diarrhoea which starts in utero. The diarrhoea is characterised by large volumes of chloride rich watery stools due to defective anion exchange transporters in the ileum and colon. We have investigated $\mathrm{CL}$ - transport in the colon using non-equilibrium rectal dialysis in children with $C C D$ and controls. 2 Caucasians had classic CCD with diarrhoea from birth (GPA.) but 4, 3 from the Arabian oulf and 1 from Nigeria presented with marked hypochloreamia (21-77mol/1), hypokalaemia, severe failure to thrive and unusually passed formed stools (GpB). Because of this feature diagnosis was delayed in $B$ and in 2/4 an erroneous diagnosis of Bartters syndrome was made. All four in B had marked secondary hyperaldosteronism plasma aldosterone 590 - >3300 pnol/1 nr 96-946; plasma renin 4300

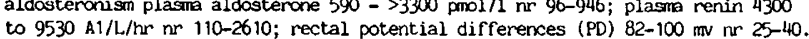
to $9530 \mathrm{~A} 1 / \mathrm{L} / \mathrm{hr} \mathrm{nr}$ 110-2610; rectal potential differences (PD) 82-100 $\mathrm{mv} \mathrm{nr} 25-40$.
Following rehydration and correction of electrolyte deficit in B pronse watery Following rehydration and correction of electrolyte deficit in B profuse watery diarrhoea developed with stool chlorides of $124-153 \mathrm{~mol} / 1$ which exceeded the sum
of $\mathrm{Na}+$ and $\mathrm{K}+\mathrm{conc}^{2}$. In A using a dialysate of $130 \mathrm{mM} \mathrm{CL}^{-} \mathrm{CL}^{-}$secretion of -70 and $-32 \mathrm{mmol} / \mathrm{min} / \mathrm{cm}^{2}$ was seen compared to absorption in controls $(n=17)+195+55$ mean $+1 \mathrm{SD}$ and $\mathrm{B}+26-+69$. In 1 of $\mathrm{B}$ the effects of varying the $\mathrm{CL}^{-}$concentration of the incubation solution on $\mathrm{CL}^{-}$fluxes was determined. 130 mM $+69,90 \mathrm{mM}-69,60 \mathrm{~mm}-107$, $30 \mathrm{~mm}-223 \mathrm{mmol} / \mathrm{min} / \mathrm{cm}^{2}$. These data suggest that $\alpha^{-}$is moving passively down chemical gradient whereas in A active secretion occurs. As diarrhoea in CCD is due to the osmotic effect of luminal $\mathrm{CL}^{-}$when $\mathrm{CL}^{-}$depletion is present it may not to the osmotic effect of luminal $C L$ when $C$ depletion is present it may not these may determine the presenting features.
PRODUCTION OF A SECOND DIARRHEAGENIC FACTOR BY $\underline{v}$.

15

CHOL.ERAE.

A. Fasano, B. Baudry, D. W. Pumplin, B.D. Tall, J.M. Ketley, J. In the last decade. MD Sch. Med., Baltimore, MD 21201 , U.S.A. ficient vaccine against cholera. When fed to volunteers, some genetically engineered mutants lacking the cholera toxin(CT) genes(CVD 101)induced mild diarrhea(1), while others(395N1)did not(2). Aim of $101)$ induced mild diarrhea( 1 , while others(395N1)did not (2). Aim of
the present study was to evaluate whether toxic factors other than the present study was to evaluate whether toxic factors other than
CT are involved in the pathogenesis of cholera. Methods: crude culCT are involved in the pathogenesis of cholera. Methods:crude cul-
ture supernatants from $\mathrm{v}$. cholerae 395 and from two similarly constructed CT- mutants(CVD̄101 and 395NI) were added to rabbit intestine stripped of serosal and muscular layers and mounted in Ussing chambers.At the end of the experiments,tissues were processed for electron microscopy. Results:a) 395 and CVD101 supernatants added to the mucosa of small intestine gave a significant increase in tissue conductance(Gt) peaking after $2 \mathrm{hrs}$. This increase caused an early increase in short circuit current(Isc) that was not related early increase in short circuit current(Isc) that was not related
to CT activity. No change in Gt was observed when 395 supernatant was added to the caecal tissue; b) 395N1 did not increase Gt for 100 min. when added to the small intestine;c)preliminary freeze-fracture data showed that tight-junctions $(t j)$ became less complex, having fewer intersections, in tissues exposed to 395 or CVDl01 supernatants, compared to those exposed to medium alone. Conclusions: 1 ) 395 and CVDI01 supernatants Induced a significant increase in Gt in rabbit small intestine, while 395N (non diarrheagenic CT- mutant) did not.Exposing caecum to 395 did not alter Gt;2) the Gt Increase was associated with morphological changes of $t j ; 3$ ) this factor may be responsible for the residual diarrhea observed in some Cr-

1.Infect. Immun. 56, 161-168(1988); 2.J.Exp.Med.168, 1487-1492(1988).
13 THE CYTOKINES INTERFERON- $\gamma$ (IFN- $\gamma$ ) AND INTER13 LEUKIN 4 (IL-4) PROMOTE TRANSCYTOSIS OF SECRET Zina Moldoveanu. Serem Frcier. John Phillios. Jiri Meslecky. The Department of Microbiology and Medicine, University of Alabama at Birmingam and the Pediatric Rescarch Laboratory, Shaare Zedek Birmingham, and the Pediatric Rescarch Laboratory, Shaare Zedek Medical Center, Jcrusalem. We have previously demonstrated that the HT-29 human coion carcinoma cell line expresses secretory
component (SC), and that cullure of these cells with IL-4 and with
IFN- $\gamma$ considerably augments the expression of SC in these cells. Our objective here was to ascertain if this is associated with increased transcytosis of SIgA across the enterocyte. Transparent Biopore membrane inserts were coated with human placental collagen matrix. The coated inserts were placed in 6 well cell cluster culture plates, $36 \mathrm{~mm}$ in diameter. On the following day, IIT-29 cells maintained in RPMI 1640 were secded in the inserts in a concentration of $10^{7} \mathrm{cells} / \mathrm{ml}$. RPMI 1640 only was placed in the outer well. Twenty four hours later the cells formed a macroscopic monolayer on the filler. INF-y and IL-4 were added in a concentration of $100 \mathrm{u} / \mathrm{ml}$ each. Forty eight hours later, $125 \mathrm{I}$ labelled monomeric IgA (mIgA) (control) and polymeric $\operatorname{IgA}(\mathrm{pIgA})$ (test) were added to the inserts. After $24 \mathrm{~h}$, total and SC-associated radioactivity were measured in the fluid of the outer wells. It was found that SC-associated radioactivity was $3 x$ higher in the $\mathrm{PIgA}$ wells than in the mIgA wells. It was also $3 x$ higher when compared to filters coated with the CaCo 2 human carcinoma cell linc, liow'll

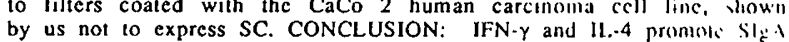

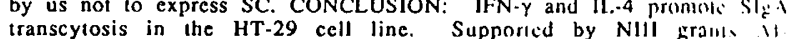
transcytosis in the HT-29 cell line. Supporled by N
18745. AI-10854. DK-28537 and GIF grant T-79-063.2/88.
IN SITU HYBRIDIZATION FOR DETFCTION OF HEPATITIS B VI16 RUS Children's S. Wirth, A. Hueter, K.M. Keller, W. Raumilann, B. Zabel Detection of hepatitis B virus (HBV)-DNA in the liver of chronic infected patients is presently the most sensitive marker of viral replication and infectivity. In situ hybridization (ISH) allows the direct visualization of HBV infected liver cells and distribution of the viral sequences. This study was done to establish ISIl and correlate the findings with conventional morkers for HBV infection.

Methods. Liver biopsies of 50 patients $\left(28 \sigma^{\circ}, 222^{\circ}\right)$ aged $0.5-20$ years (mean 10.3) with various histological diagnoses were lested by ISH. The HBV-DNA probe was labeled by nick translation with ${ }^{35} \mathrm{~S}-\mathrm{CTP}$ to a specific activity of $3-5 \times 10^{8} \mathrm{cpm} / \mathrm{hg} \mathrm{DNA}$.

Results. HBV-DNA/mRNA could be demonstrated in 38 patients, 12 were negative. Distribution of the grains was homogenous, inhomogenous with focal patches and focal. $33 / 38$ children with IllBeAg and $4 / 11$ with anti-liBe were positive by ISil, 5/38 with HBCAg and $7 / 11$ with anti-l1Be remained negative. $17 / 23 \mathrm{HBsAg}$ carriers with positive IIBV-DNA/mRNA by ISH were positive for IIBCAy in the liver positive $31 / 36$ had free HBV-DNA in Southern blot hybridization. and $31 / 36$ had free HBV-DNA in Southern blot hybridization.
Conclusions. Our results indicate that hepatitis B viral genomes can reliably be detecled by in silu hybridization. Although there is a good correlation to other HBV markers (HBeAg $\mathrm{HBcAg}$ and Southern blot hybridization) ISH may represent a more sensitive method to prove viral replication and infectivity. 